\title{
Stent Thrombosis after Endovascular Treatment of Iliofemoral or Caval Veins in Patients with Postthrombotic Syndrome
}

\author{
Andrea Cervi $^{1}$ James D. Douketis ${ }^{1}$ \\ ${ }^{1}$ Department of Medicine, McMaster University, Hamilton, \\ Ontario, Canada \\ Thromb Haemost 2019;119:1897-1899.
}

Address for correspondence James D. Douketis, MD, FRCP, St. Joseph's Healthcare Hamilton, Room F-544, 50 Charlton Avenue East, Hamilton, Ontario L8N 4A6, Canada (e-mail: jdouket@mcmaster.ca).
Following acute deep vein thrombosis, 20 to $50 \%$ of patients will develop the postthrombotic syndrome (PTS), despite optimal antithrombotic therapy. ${ }^{1}$ Venous remodeling underlies the pathophysiology of PTS and clinically manifests as painful leg edema, hyperpigmentation, venous ectasia, and, sometimes, ulcers ( $\mathbf{- F i g . 1}$ ). ${ }^{2}$ PTS is associated with a significant financial and morbidity burden, and reduced patient-reported health quality of life measures. ${ }^{1,2}$ There have been considerable efforts in improving the prediction $^{3-5}$ and prevention ${ }^{6-8}$ of PTS in recent years. Indeed, the prevention of PTS has focused on use of external compression stockings, different forms of antithrombotic therapy, anti-inflammatory drugs, and acute endovascular interventions such as catheter-directed thrombolysis, all of which have provided conflicting results about efficacy. Similarly, the treatment of established PTS is unclear given the lack of well-designed studies and evidence-based management strategies.

A subset of patients with severe PTS may derive benefit from endovascular therapy, namely those with chronic iliac vein obstruction. Image-guided, catheter-based stent placement in patients with residual iliofemoral venous obstruction has been associated with high technical success rates and patient-reported symptom relief. ${ }^{9,10}$ However, the overall quality of evidence for important clinical outcomes, including complications, appears to be low. Stent thrombosis (ST) is a particularly feared complication of endovascular venous stenting that may limit its uptake in patients with PTS. However, details relating to the timing of ST following stent insertion, risk factors for ST development and impact of different antithrombotic regimens on ST have not been well characterized.

Against this background, Sebastian et al conducted a subgroup analysis of 136 patients with PTS and venous stent implants enrolled in the Swiss Venous Stent Registry to shed light on the timing, the incidence, and risk factors for ST during and after antithrombotic therapy. ${ }^{11}$

Early discontinuation of antithrombotic therapy occurred more commonly in patients who were young, female, and had May-Thurner syndrome, whereas patients with more severe PTS at baseline continued anticoagulation. These patients were more often obese and had a history of recurrent venous thrombotic events. Antithrombotic therapy was given to $96 \%$ of patients for at least 3 months; $32 \%$ completed a median duration of 12 months of treatment. Approximately half of patients (46\%) were started on low-molecular-weight heparin after stent insertion before transitioning to an oral agent, most often a direct oral anticoagulant.

Continued antithrombotic therapy did not appear to impact on the development of ST or recurrent venous thrombotic events. Similarly, the type of oral anticoagulant (i.e., vitamin $\mathrm{K}$ antagonist vs. direct oral anticoagulant) did not influence ST rates. ST occurred in $20 \%$ of patients, at a median of 96 days poststent placement (median $=89$ days, interquartile range [IQR] 13-136 in patients who continued antithrombotic therapy, median $=289$ days, IQR 69-900 in patients who stopped treatment). The cumulative incidence of ST was greatest within the first 6 months following stent insertion (13.7\%, 95\% confidence interval [CI]: 7.8-19.6).

Risk factors for development of ST were: age $<40$ years (hazard ratio $[\mathrm{HR}]=2.26,95 \% \mathrm{CI}: 1.03-4.94$ ), stent insertion distal to the common femoral vein ( $\mathrm{HR}=3.03$; $95 \% \mathrm{CI}$ : 1.28 7.19 ), and the presence of postthrombotic femoral inflow veins ( $\mathrm{HR}=2.92$; 95\% CI: 1.36-6.25). Interestingly, a diagnosis of May-Thurner syndrome was associated with a reduced risk for ST $(\mathrm{HR}=0.37 ; 95 \% \mathrm{CI}$ : 0.15-0.91).

Endovascular stent placement was highly efficacious in maintaining venous recanalization, with a 99\% primary treatment success rate. Moreover, stent placement was associated with improvements in the Villalta score (median

\section{received}

September 16, 2019

accepted

September 16, 2019 (c) 2019 Georg Thieme Verlag KG Stuttgart · New York
DOI https://doi.org/ 10.1055/s-0039-1700808. ISSN 0340-6245. 


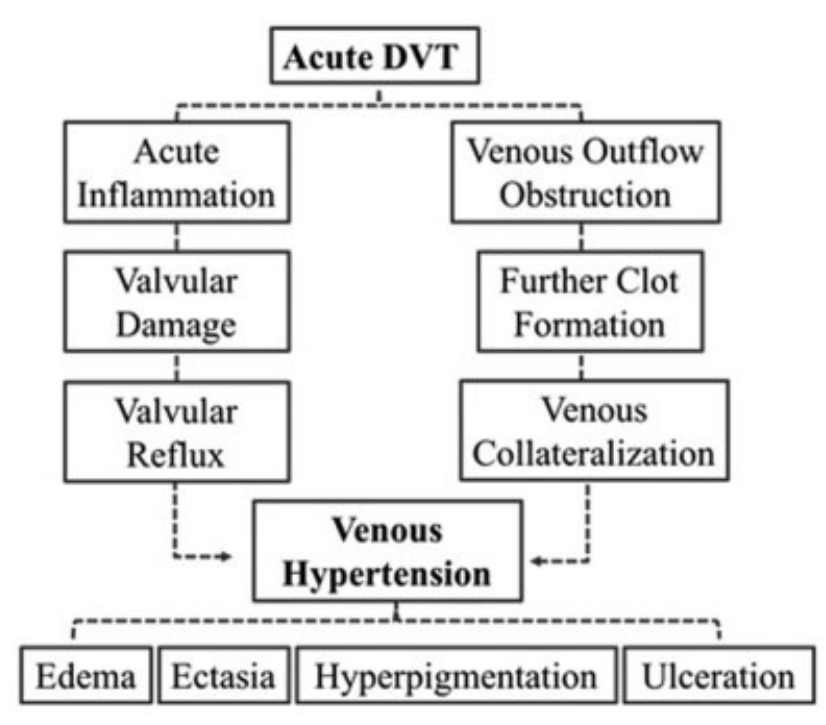

Fig. 1 Pathophysiology of postthrombotic syndrome.

5-point reduction, IQR 1-7) and revised venous clinical severity score (median 3-point reduction, IQR 0-6), with $66 \%$ of patients free from PTS symptoms at the time of last follow-up. Bleeding events complicated $15 \%$ of patients, with no major difference among patients who continued antithrombotic therapy (14\%) and those who did not (16\%).

The main findings from this study help to address the paucity of data regarding clinical outcomes and complications of endovascular stent placement for PTS. The majority of venous stent thrombosis occurred during anticoagulant therapy, although the quality of anticoagulation, namely time in therapeutic range while receiving warfarin, was not reported. Moreover, patients receiving continued anticoagulant therapy were at higher baseline risk of thrombosis compared with those who stopped anticoagulation early, making it difficult to assess the comparative efficacy of anticoagulation in preventing ST in PTS. Nonetheless, these findings highlight the role of alternative risk factors such as younger patient age, distal stent insertion, and postthrombotic venous collateralization in ST development. Whether patients with these risk factors warrant more aggressive monitoring for ST or combination anticoagulant-antiplatelet therapy remains uncertain.

Antithrombotic management strategies following endovascular venous stent insertion are inconsistent and lack uniform consensus among major societal guidelines. ${ }^{12-14}$ Moreover, the ideal anticoagulant regimen is uncertain. Although the results from the study of Sebastian et al suggest no difference with treatment using low-molecular-weight heparin, vitamin $\mathrm{K}$ antagonists, or direct oral anticoagulants, the study sample size was small and application of the study findings are limited to patients receiving self-expanding nitinol venous stents. Whether these findings can be applied to patients receiving alternative types of endovascular venous stents (i.e., stainless steel, covered stent grafts, etc.) also is uncertain. Adjunct antiplatelet therapy was used in $15 \%$ of patients in this study but details relating to the type, dose, duration, anticoagulant pairing, and specific outcome measures relative to patients receiving anticoagulant therapy alone were not reported.
While this study represents a step forward toward defining outcomes in patients with PTS and venous stents, several important questions remain. Results of the randomized, controlled, open-label ARIVA trial (EudraCT registration number: 2019-001723-12) comparing aspirin and rivaroxaban to rivaroxaban alone in patients with endovascular venous stents will be key in determining the optimal antithrombotic strategy in patients with venous stents. Moreover, the ongoing C-TRACT trial (\#NCT03250247), a randomizedcontrolled trial evaluating the role of endovascular therapy in patients with iliac obstructive PTS will help to shed further light on the comparative efficacy and safety of endovascular venous stenting for treatment of PTS. The management of PTS remains one of the most challenging situations in patients with thrombotic vascular disease. Efforts by Sebastian et al aimed at improving patient outcomes are welcome but more work is needed.

Conflict of Interest

J.D.D. reports personal fees from Pfizer, Sanofi, Leo Pharma, Bristol Myers Squibb, Janssen, The Merck Manual, Up-to-date, Portola, outside the submitted work. A.C. has nothing to disclose.

\section{References}

1 Kahn SR. How I treat postthrombotic syndrome. Blood 2009;114 (21):4624-4631

2 Rabinovich A, Kahn SR. How I treat the postthrombotic syndrome. Blood 2018;131(20):2215-2222

3 Dronkers CEA, Mol GC, Maraziti G, et al. Predicting post-thrombotic syndrome with ultrasonographic follow-up after deep vein thrombosis: a systematic review and meta-analysis. Thromb Haemost 2018;118(08):1428-1438

4 Méan M, Limacher A, Alatri A, Aujesky D, Mazzolai L. Derivation and validation of a prediction model for risk stratification of post-thrombotic syndrome in elderly patients with a first deep vein thrombosis. Thromb Haemost 2018;118(08):14191427

5 Amin EE, van Kuijk SMJ, Joore MA, Prandoni P, Ten Cate H, Ten Cate-Hoek AJ. Development and validation of a practical two-step prediction model and clinical risk score for post-thrombotic syndrome. Thromb Haemost 2018;118(07):1242-1249

6 Haig Y, Enden T, Grøtta O, et al; CaVenT Study Group. Postthrombotic syndrome after catheter-directed thrombolysis for deep vein thrombosis (CaVenT): 5 -year follow-up results of an open-label, randomised controlled trial. Lancet Haematol 2016;3 (02):e64-e71

7 Vedantham S, Goldhaber SZ, Julian JA, et al; ATTRACT Trial Investigators. Pharmacomechanical catheter-directed thrombolysis for deep-vein thrombosis. N Engl J Med 2017;377(23): 2240-2252

8 Rabinovich A, Kahn SR. The postthrombotic syndrome: current evidence and future challenges. JThromb Haemost 2017;15(02): 230-241

9 Razavi MK, Jaff MR, Miller LE. Safety and effectiveness of stent placement for iliofemoral venous outflow obstruction: systematic review and meta-analysis. Circ Cardiovasc Interv 2015;8 (10):e002772

10 Qiu P, Zha B, Xu A, et al. Systematic review and meta-analysis of iliofemoral stenting for post-thrombotic syndrome. Eur J Vasc Endovasc Surg 2019;57(03):407-416

11 Sebastian T, Spirk D, Engelberger R, et al. Incidence of stent thrombosis after endovascular treatment of iliofemoral or caval 
veins in patients with the postthrombotic syndrome. Thromb Haemost 2019;119(12):2064-2073

12 Kearon C, Akl EA, Ornelas J, et al. Antithrombotic therapy for VTE disease CHEST guideline and Expert Panel Report. Chest 2016;149 (02):315-352

13 Jaff MR, McMurtry MS, Archer SL, et al; American Heart Association Council on Cardiopulmonary, Critical Care, Perioperative and Resuscitation; American Heart Association Council on Peripheral Vascular Disease; American Heart Associ- ation Council on Arteriosclerosis, Thrombosis and Vascular Biology. Management of massive and submassive pulmonary embolism, iliofemoral deep vein thrombosis, and chronic thromboembolic pulmonary hypertension: a scientific statement from the American Heart Association. Circulation 2011; 123(16): 1788-1830

14 Milinis K, Thapar A, Shalhoub J, Davies AH. Antithrombotic therapy following venous stenting: international Delphi consensus. Eur J Vasc Endovasc Surg 2018;55(04):537-544 